\title{
Abstract
}

\section{Modified Unilateral Buccinator Myomucosal Flap technique for Correction of Velopharyngeal Insufficiency due to insufficient Palatal length and mobility}

\author{
Herath $\mathbf{M}^{1}$, Dias $\mathrm{DK}^{1}$, Perera $\mathrm{B}^{2}$ \\ ${ }^{1}$ Teaching Hospital, Karapitiya, ${ }^{2}$ Faculty of Medicine, University of Ruhuna
}

Key words: buccinator mucosal flap, velopharyngeal insufficiency

\section{Objective}

To assess the outcome of palate lengthening by modified unilateral myomucosal buccinator flap for velopharyngeal insufficiency (VPI) in terms of speech intelligibility and soft palate length.

Methods

The study setting was the multi-disciplinary regional cleft center of southern Sri Lanka based at Teaching Hospital, Karapitiya, Galle. A consecutive sample of 60 patients who underwent the modified unilateral buccinator myomucosal flap procedure between 2004 and 2010 were reviewed retrospectively. All patients were assessed six months after the surgery. Palatal length was recorded by direct measurement in millimeters pre and post operatively. Speech was evaluated blindly by a speech therapist using pre and post operative digital speech records.

\section{Results}

Mean increase of palate length after insertion of the buccinator flap was $7.61 \pm 1.85$ $\mathrm{mm}$ with a range of $4 \mathrm{~mm}$ to $13 \mathrm{~mm}$. Marked and statistically significant $(p<0.001)$ improvement of hypernasality, nasal emission and consonant production errors were observed after surgery. No statistically significant worsening of hypo-nasality was observed.

\section{Conclusion}

This paper demonstrates that with the proposed modifications to the unilateral myomucosal buccinator flap, we can achieve satisfactory results in par with or superior to the bilateral myomucosal buccinator flap technique in patients with VPI due to short palate length and/or decrease mobility of soft palate. 